\title{
Nonlinear vibration characteristics of a flexible blade with friction damping due to tip-rub
}

\author{
Dengqing $\mathrm{Cao}^{\mathrm{a}, *}$, Xiaochun Gong ${ }^{\mathrm{a}}$, Dong $\mathrm{Wei}^{\mathrm{a}}$, Shiming $\mathrm{Chu}^{\mathrm{a}}$ and Ligang Wang ${ }^{\mathrm{b}}$

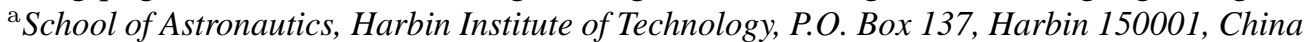 \\ ${ }^{\mathrm{b}}$ School of Science, Harbin Engineering University, Harbin 150001, China
}

Received 10 February 2010

Revised 20 June 2010

\begin{abstract}
An approximate approach is proposed in this paper for analyzing the two-dimensional friction contact problem so as to compute the dynamic response of a structure constrained by friction interfaces due to tip-rub. The dynamical equation of motion for a rotational cantilever blade in a centrifugal force field is established. Flow-induced distributed periodic forces and the internal material damping in the blade are accounted for in the governing equation of motion. The Galerkin method is employed to obtain a three-degree-of-freedom oscillator with friction damping due to tip-rub. The combined motion of impact and friction due to tip-rub produced a piecewise linear vibration which is actually nonlinear. Thus, a complete vibration cycle is divided into successive intervals. The system possesses linear vibration characteristic during each of these intervals, which can be determined using analytical solution forms. Numerical simulation shows that the parameters such as gap of the tip and the rotational speed of the blades have significant effects on the dynamical responses of the system. Finally, the nonlinear vibration characteristics of the blade are investigated in terms of the Poincare graph, and the frequency spectrum of the responses and the amplitude-frequency curves.
\end{abstract}

Keywords: Nonlinear vibration, flexible blade, friction damping, tip-rub, impact

\section{Introduction}

In the turbine jet engine industry, one of the main failure problems of the turbine engine blades can be attributed to high cyclic fatigue caused by large resonance stress. Preventing turbo-machinery blade failures has been an important issue in the turbine engine. Dry friction damper, a device to reduce vibration amplitude and increase blade damping, is proved to be a highly effective way to dissipate energy of vibration. The friction damper absorbs vibration energy through the friction between the adjacent shrouded blades. The interface friction may include slip, stuck and separations, which lead to very complex contact kinematics. This leads it a difficult task to predict its dynamic response accurately.

Because of the strong nonlinear nature, approximation methods or numerical integration methods are adopted to deal with the system in most investigations. The simplest interface model is the Coulomb friction model where contact points do not move with respect to each other unless the friction force exceeds a critical limit. Den Hartog [1] was one of the earliest scientists to study the dynamic behavior of structures with Coulomb friction. The exact solution of steady state motion is obtained through a single-DOF dry fiction oscillator. This method was developed

\footnotetext{
* Corresponding author: Professor Dengqing Cao, Tel.: +86 451 86414479; Fax: +86 451 86414479; E-mail: dqcao@ hit.edu.cn.
} 
to a two-DOF system in [2,3] and multiple-DOF systems in [4,5] later. In these works, authors used harmonic balance method in combination with a structural modification approach in order to perform simulations [6,7]. These investigations were based on a kind of dry friction model, which is called "macro-slip" model and is widely used [8]. The friction interfaces are modeled as a rigid body, and include either slip state or stick state. Suppose that the normal load of all points of the contact interface are equal and small, can be seen as a single point of contact model with a point to replace the entire contact interface, and all points of interface sticking or slipping at the same time. Iwan [9] proposed the famous bi-linear hysteretic restoring force model. Menq and Griffin [10,11] perfected the model proposed in [9] and developed a finite element model to demonstrate the limitations of the model in [9]. Ding Qian et al. [12,13] divided a complete vibration cycle into four successive intervals to determine the steady state response of the blade. Nan et al. [14] formulated a simple two-DOF system in terms of the Sgn contact model. Numerical results are given in [14] to analyze effects of parameters on nonlinear dynamic characteristics of the system.

On the other hand, Menq et al. [15] developed the micro-slip method a few years later. The theory suggests that the friction interface is an elastic body if the normal load acting on the interface is high; it is capable of modeling partial slip before all point slip. One-dimensional motion is not valid enough, especially when there is coupling between motions of the system in more than one direction. Griffin and Menq [16-19] investigated a two-dimensional model by assuming points of contact move in a circular path and dividing the contact into parallel connection of some small macro-slip model elements. Yang et al. [20] and Chen and Menq [21] investigated the three-dimensional shroud contact kinematics of a shrouded blade system. They assumed blade motion has three components: axial, tangential, and radial components. The connected states have been explained easier by the model, but too much parametric hypothesis affect the calculation accuracy of steady state response. By analyzing the micro-slip method and the macro-slip method contrastively, Ciğeroğlu and Özgüven [22] indicated that the micro-slip model can provide more accurate results by applying a quasi-linearization technique. They proposed a new model about all blades around of the disk, which developed the micro-slip method and validated feasibility of the model.

In this paper, the basic dynamical equation of motion for a rotational cantilever flexible blade in a centrifugal force field is established to simulate the dynamical responses of the blade of an aero-engine rotor. Non-proportional viscous damping model is used to formulate the effect of frictional constraint at the shroud interfaces. The dynamical equation of the system is established and the Galerkin method is applied to discretize the partial differential equations to a 3-DOF system so as to compute the dynamic responses of the aero-engine blade constrained by friction interfaces due to tip-rub. Then the differential equations of motion with 3-DOF are numerically solved by using the RungeKutta method. Several results related to damped vibration characteristics of the blade with regard to rotational speed and gap are numerically obtained respectively.

\section{Mathematical modeling formulation}

\subsection{The governing equations of motion}

For the sake of convenience, a blade of an aero-engine rotor is simplified as a continuous cantilever in a centrifugal force field. A rotating blade with tip-rub is considered to be a uniform and initially straight cantilever, constrained at the free end by springs and friction dampers with a gap $\Delta$ as sketched in Fig. 1. With the tip-rub, when the lateral displacement is larger than the width of the gap on tip of the blade, a normal pressure and a friction force are imposed.

The equation of motion of the blade can readily be derived by considering the equilibrium of forces and moments acting on the differential segment of the blade with the length of $d x$. Summing all forces in transversal direction leads to the first dynamic equilibrium relationship with respect to transverse displacements $v(x, t)$

$$
\begin{gathered}
Q-f \frac{\partial v}{\partial x}-\left[Q+\frac{\partial Q}{\partial x} d x-f \frac{\partial v}{\partial x}-\frac{\partial\left(f \frac{\partial v}{\partial x}\right)}{\partial x} d x\right]-c \frac{\partial v}{\partial t} d x \\
+\left[F_{a}(t)-\left(N \cos \gamma+F_{f} \sin \gamma\right) \delta(x-l)\right] d x=\rho A d x \frac{\partial^{2} v}{\partial t^{2}}
\end{gathered}
$$




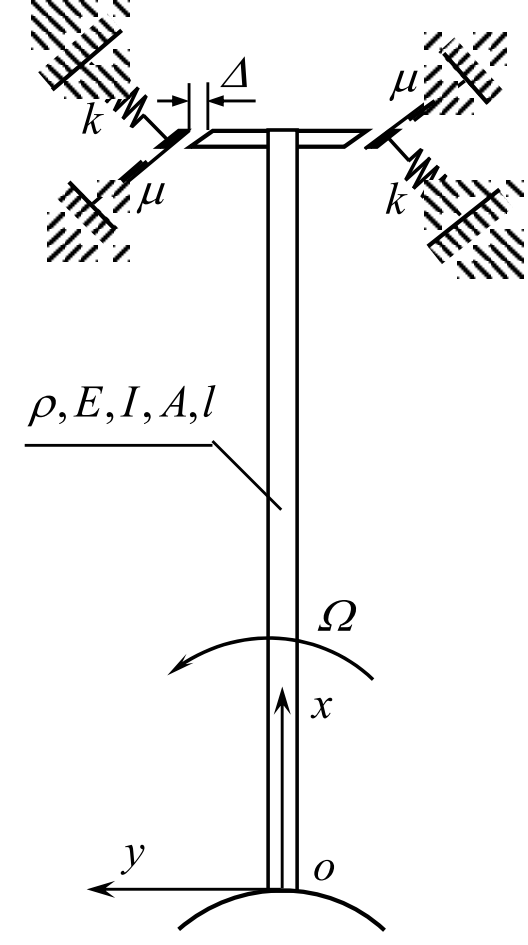

Fig. 1. Rotating blade with tips of an aero-engine rotor.

where $Q$ is the shear force acting on the cross-section, $\rho$ is the mass density, $A$ is the cross-section area, $l$ is the length of the blade, $c$ is the viscous damping coefficient, and $\Omega$ is the rotating speed of the rotor. The axial load $f(x)$ can be written as

$$
f(x)=\frac{1}{2} \rho A \Omega^{2}\left(l^{2}-x^{2}\right) .
$$

The second equilibrium relationship is obtained by summing moments acting on the differential segment. This leads to the relationship between shear and moment.

Introducing the basic moment-curvature relationship, we have

$$
Q=\frac{\partial M}{\partial x}=\frac{\partial}{\partial x}\left(E I \frac{\partial^{2} v}{\partial x^{2}}\right)
$$

in which $M$ is the moment acting on the cross-section, and $E I$ is the flexural rigidity.

The aerodynamic forces acting on the blade of an aero-engine is very complex. For simplicity, we assume that the blade is excited by a distributed periodic aerodynamic load. Then, the governing equation of motion for a single rotational cantilever flexible blade can be formulated from Eqs (1), (2) and (3) as

$$
\begin{aligned}
E I \frac{\partial^{4} v}{\partial x^{4}}+\rho A \frac{\partial^{2} v}{\partial t^{2}}+c \frac{\partial v}{\partial t} & =-\rho A \Omega^{2} x \frac{\partial v}{\partial x}+\frac{1}{2} \rho A \Omega^{2}\left(l^{2}-x^{2}\right) \frac{\partial^{2} v}{\partial x^{2}} \\
& +F_{a}(t)-\left(N \cos \gamma+F_{f} \sin \gamma\right) \delta(x-l),
\end{aligned}
$$

where, the external periodic aerodynamic force is defined as $F_{a}=F_{a 0} \sin (3 \Omega t)$ and $F_{a 0}$ is the amplitude of the external load. $\gamma$ is the contact interface angle as shown in Fig. 2(a), $N$ and $F_{f}$ denote the normal and friction force at the tip-rub as shown in Fig. 2(b). The collision pressure $N$ and the friction force $F_{f}$ imposed on the tip of the blade are respectively described as

$$
N=\left\{\begin{array}{cl}
k(v(l)-\Delta) \cos \gamma, & v(l)>\Delta, \\
0, & -\Delta<v(l)<\Delta, \\
k(v(l)+\Delta) \cos \gamma, & v(l)<-\Delta,
\end{array}\right.
$$




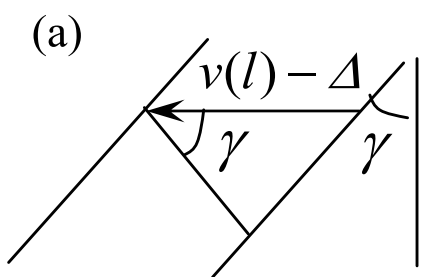

(b)

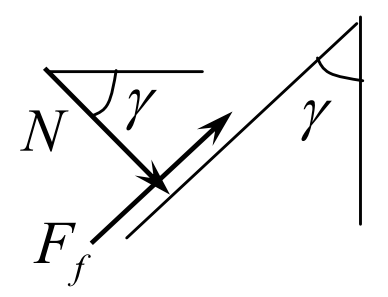

Fig. 2. The geometrical relations of the displacements (a) and forces (b) on the contact interface.

where $\Delta$ is the gap between two adjacent tips of the blades as shown in Fig. 1, $k$ is the elastic shear stiffness between two adjacent contacting interfaces, $v(l)$ is the transverse displacement at the free end of the blade, and

$$
F_{f}= \begin{cases}\mu_{k} N, & \dot{v}(l)>0, \\ -\mu_{s} N \leqslant g(t) \leqslant \mu_{s} N, & \dot{v}(l)=0, \\ -\mu_{k} N, & \dot{v}(l)<0,\end{cases}
$$

in which $\mu_{k}$ is the dynamic friction coefficient, $\mu_{s}$ is the static friction coefficient and $\dot{v}(l)$ is the relative sliding velocity between the contacting interfaces.

Putting the Eq. (4) in dimensionless forms, we obtain the governing equation of motion

$$
\bar{v}^{(4)}+\beta^{2} \ddot{\bar{v}}+\zeta \beta \dot{\bar{v}}=-\beta^{2} \bar{v}^{(1)} \bar{x}+\frac{1}{2} \beta^{2}\left(1-\bar{x}^{2}\right) \bar{v}^{\prime \prime}+\eta\left[F_{a 0} \sin (3 \tau)-\left(N \cos \gamma+F_{f} \sin \gamma\right) \delta(\bar{x}-1)\right],
$$

where

$$
\begin{aligned}
& \bar{v}=\frac{v}{l}, \quad \bar{x}=\frac{x}{l}, \quad \tau=\Omega t, \quad \bar{\omega}=\sqrt{\frac{E I}{\rho A l^{4}}} \\
& \beta=\frac{\Omega}{\bar{\omega}}, \quad \zeta=\frac{c}{\rho A \bar{\omega}}, \quad \eta=\frac{l^{3}}{E I} .
\end{aligned}
$$

Accordingly, the normal force in Eq. (5) can be written as the following form

$$
N=\left\{\begin{array}{cl}
k l[\bar{v}(1)-\bar{\Delta}] \cos \gamma, & \bar{v}(\bar{x})>\bar{\Delta} \\
0, & -\bar{\Delta}<\bar{v}(\bar{x})<\bar{\Delta}, \quad \bar{\Delta}=\frac{\Delta}{l} . \\
k l[\bar{v}(1)+\bar{\Delta}] \cos \gamma, & \bar{v}(\bar{x})<-\bar{\Delta}
\end{array}\right.
$$

The accompanying boundary conditions are those of a fixed-free beam

$$
\bar{v}(0, \tau)=\bar{v}^{\prime}(0, \tau)=\bar{v}^{\prime \prime}(1, \tau)=\bar{v}^{\prime \prime \prime}(1, \tau)=0 .
$$

\subsection{Galerkin discretization}

Applying the Galerkin method and introducing the first $m$ terms of bending modes, the dimensionless transverse displacement variable is expanded as

$$
\bar{v}(\bar{x}, \tau)=\sum_{i=1}^{m} \phi_{i}(\bar{x}) q_{i}(\tau)
$$

For approximate solution, we take the orthonormal sets of eigen-functions for a cantilever as the modal functions for the rotating blade, i.e.,

$$
\phi_{i}(\bar{x})=\cosh \lambda_{i} \bar{x}-\cos \lambda_{i} \bar{x}-\frac{\cosh \lambda_{i}+\cos \lambda_{i}}{\sinh \lambda_{i}+\sin \lambda_{i}}\left(\sinh \lambda_{i} \bar{x}-\sin \lambda_{i} \bar{x}\right) .
$$

Substituting Eq. (10) into Eq. (7) and orthogonalizing the latter with respect to the set $\varphi_{j}(\bar{x})$, we obtain 


$$
\begin{aligned}
\sum_{i=1}^{m} q_{i}(\tau) \int_{0}^{1} \varphi_{j} \varphi_{i}^{(4)}(\bar{x}) d \bar{x}+ & \ddot{q}_{j}(\tau) \beta^{2} \int_{0}^{1} \varphi_{j}^{2}(\bar{x}) d \bar{x}+\zeta \beta \dot{q}_{j}(\tau) \int_{0}^{1} \varphi_{j}^{2}(\bar{x}) d \bar{x} \\
& =-\beta^{2} \sum_{i=1}^{m} q_{j}(\tau) \int_{0}^{1} \varphi_{i}^{\prime}(\bar{x}) \varphi_{j}(\bar{x}) \bar{x} d \bar{x} \\
& +\frac{1}{2} \beta^{2} \sum_{i=1}^{m} q_{i}(\tau) \int_{0}^{1}\left(1-\bar{x}^{2}\right) \varphi_{j} \varphi_{i}^{\prime \prime}(\bar{x}) d \bar{x} \\
& +\eta\left[F_{a 0} \sin (3 \tau) \int_{0}^{1} \varphi_{j} d \bar{x}-\left(N \cos \gamma+F_{f} \sin \gamma\right) \cdot \varphi_{j}(1)\right], \\
& (j=1,2, \cdots, m) .
\end{aligned}
$$

Then the governing differential equation of motion can be formulated as

$$
M \ddot{q}+C \dot{q}+K q=F,
$$

where $q=\left\{q_{1}, \ldots, q_{m}\right\}^{T}$ is the displacement vector with independent variables. For the sake of simplifying, we take $m=3$. Then

$$
\begin{aligned}
M & =\beta^{2} \operatorname{diag}\left[\begin{array}{lll}
\int_{0}^{1} \phi_{1}^{2} d \bar{x} & \int_{0}^{1} \phi_{2}^{2} d \bar{x} & \int_{0}^{1} \phi_{3}^{2} d \bar{x}
\end{array}\right], \\
C & =\zeta \beta \operatorname{diag}\left[\begin{array}{lll}
\int_{0}^{1} \phi_{1}^{2} d \bar{x} & \int_{0}^{1} \phi_{2}^{2} d \bar{x} & \int_{0}^{1} \phi_{3}^{2} d \bar{x}
\end{array}\right], \\
K & =\left[\begin{array}{ccc}
\int_{0}^{1} \phi_{1} \phi_{1}^{(4)} d \bar{x} & \int_{0}^{1} \phi_{1} \phi_{2}^{(4)} d \bar{x} & \int_{0}^{1} \phi_{1} \phi_{3}^{(4)} d \bar{x} \\
\int_{0}^{1} \phi_{2} \phi_{1}^{(4)} d \bar{x} & \int_{0}^{1} \phi_{2} \phi_{2}^{(4)} d \bar{x} & \int_{0}^{1} \phi_{2} \phi_{3}^{(4)} d \bar{x} \\
\int_{0}^{1} \phi_{3} \phi_{1}^{(4)} d \bar{x} & \int_{0}^{1} \phi_{3} \phi_{2}^{(4)} d \bar{x} & \int_{0}^{1} \phi_{3} \phi_{3}^{(4)} d \bar{x}
\end{array}\right] .
\end{aligned}
$$

The external loads on the right hand side of the equations are as follows

$$
F=\left[A_{1} \beta^{2} q+\frac{\beta^{2}}{2} A_{2} q+A_{3} F_{a 0} \sin (3 \tau)-A_{4}\left(N \cos \gamma+F_{f} \sin \gamma\right)\right],
$$

where

$$
\begin{aligned}
& A_{1}=\left[-\int_{0}^{1} \phi_{i}^{\prime}(\bar{x}) \phi_{j}(\bar{x}) \bar{x} d \bar{x}\right]_{3 \times 3}, \quad A_{2}=\left[\int_{0}^{1}\left(1-\bar{x}^{2}\right) \phi_{i}^{\prime \prime} \phi_{j} d \bar{x}\right]_{3 \times 3}, \\
& A_{3}=\eta \int_{0}^{1}\left\{\begin{array}{c}
\phi_{1} \\
\phi_{2} \\
\phi_{3}
\end{array}\right\} d \bar{x}, \quad A_{4}=\eta\left\{\begin{array}{l}
\phi_{1}(1) \\
\phi_{2}(1) \\
\phi_{3}(1)
\end{array}\right\}, \quad q=\left\{\begin{array}{c}
q_{1} \\
q_{2} \\
q_{3}
\end{array}\right\}, \quad \dot{q}=\left\{\begin{array}{c}
\dot{q}_{1} \\
\dot{q}_{2} \\
\dot{q}_{3}
\end{array}\right\}, \\
& \bar{v}(1)=\sum_{i=1}^{3} \phi_{i}(1) q_{i}(\tau) .
\end{aligned}
$$

\section{Numerical results and discussion}

To demonstrate the efficiency and convergence of the proposed method to solve the forced vibration problem, several results related to damped vibration characteristics of the blade with regard to the rotational speed $\Omega$, the tip gap $\Delta$ and elastic coefficient $k$ are numerically obtained respectively. The initial tip gap is chosen to be $\Delta=0.02 \mathrm{~mm}$ and the initial elastic coefficient between tips is $k=1.0 \times 10^{4} \mathrm{~N} / \mathrm{mm}$. The frequency of the external periodic aerodynamic force is assumed to be 3 times the rotational frequency (see Eq. (7)). A set of typical parameters are 
Table 1

Material constants and geometrical parameters for the system

\begin{tabular}{clcc}
\hline Sign & Description & Value & Unit \\
\hline$E I$ & flexural rigidity & 343 & $\mathrm{~N} \cdot \mathrm{m}$ \\
$A$ & cross-section area & $4.2 \times 10^{-4}$ & $\mathrm{~m}^{2}$ \\
$\rho$ & mass density & 7800 & $\mathrm{Kg} / \mathrm{m}^{3}$ \\
$l$ & length of the blade & 150 & $\mathrm{~mm}$ \\
$c$ & viscous damping coefficient & 29.8 & $\mathrm{~N} \cdot \mathrm{s} / \mathrm{m}$ \\
$b$ & width of the blade & 60 & $\mathrm{~mm}$ \\
$h$ & thickness of the blade & 7 & $\mathrm{~mm}$ \\
$k$ & elastic coefficient between tips & $1 \sim 4 \times 10^{4}$ & $\mathrm{~N} / \mathrm{mm}$ \\
$\mu$ & rub coefficient & 0.33 & \\
$\Delta$ & gap between the blade tips & $0.02 \sim 0.2$ & $\mathrm{~mm}$ \\
$\gamma$ & contact angle between tips & $\pi / 6$ & \\
$n$ & rotational speed of the blade & $1000 \sim 10000$ & $\mathrm{rpm}$ \\
\hline
\end{tabular}

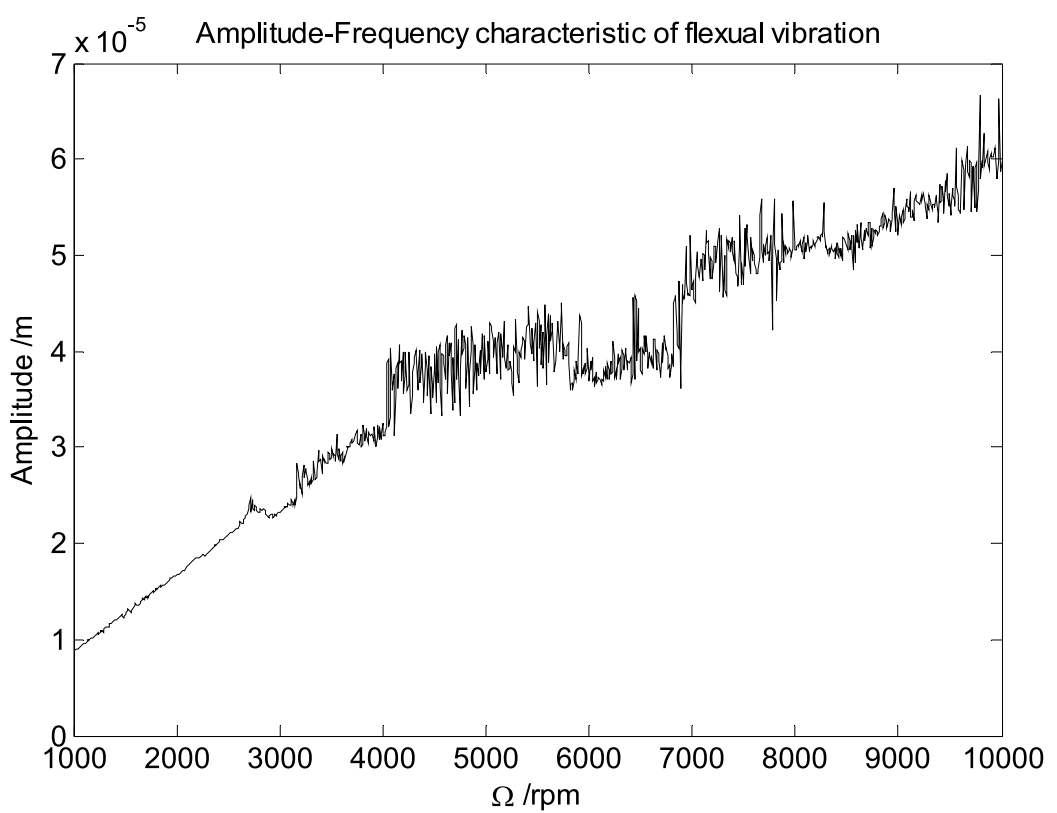

Fig. 3. Amplitude frequency characteristics of the flexural vibration of the blade.

chosen and listed in Table 1. The first three natural frequencies of the blade can be easily obtained as $245.5 \mathrm{~Hz}$, $1594.8 \mathrm{~Hz}$ and $4465.9 \mathrm{~Hz}$.

The responses of the rotating blade-tip system with the variation of the rotational speed from $1000 \mathrm{rpm}$ to 10000 rpm are worked out to illustrate the effects of friction damping on the dynamic behavior of system. Figure 3 presents the amplitude frequency characteristics of the system when the tip gap $\Delta$ is fixed to $0.02 \mathrm{~mm}$. It can be observed from Fig. 3 that the displacement of the flexural vibration is getting larger with the increase of the rotational speed under a certain tip gap $\Delta$. When the rotational speed is less than $2410 \mathrm{rpm}$, it can be observed that the displacement of the blade end is smaller than the initial gap $\Delta=0.02 \mathrm{~mm}$. That is to say the vibration amplitude of the blade end is not large enough to collide with the tip. When the rotational speed is greater than $2410 \mathrm{rpm}$, the displacement of the blade end is larger than the minimal tip gap, which indicates that the collision between the blade end and the tip will set to work to decrease the vibration. Figure 4 shows the response of the blade with $\Delta=0.1 \mathrm{~mm}$ at the rotating speed $n=2000 \mathrm{rpm}$ and $n=9000 \mathrm{rpm}$ respectively by the time history, phase diagram, Poincaré map and spectrum analysis. The motions of the system with and without collision are steady periodic and quasi-periodic motion, respectively.

Figure 5 presents the relationship between the amplitude of dynamical responses at the free end versus the tip gap and the rotational speed of the blade. The tip gap varies from $0.02 \mathrm{~mm}$ to $0.2 \mathrm{~mm}$ and the rotational speed varies 

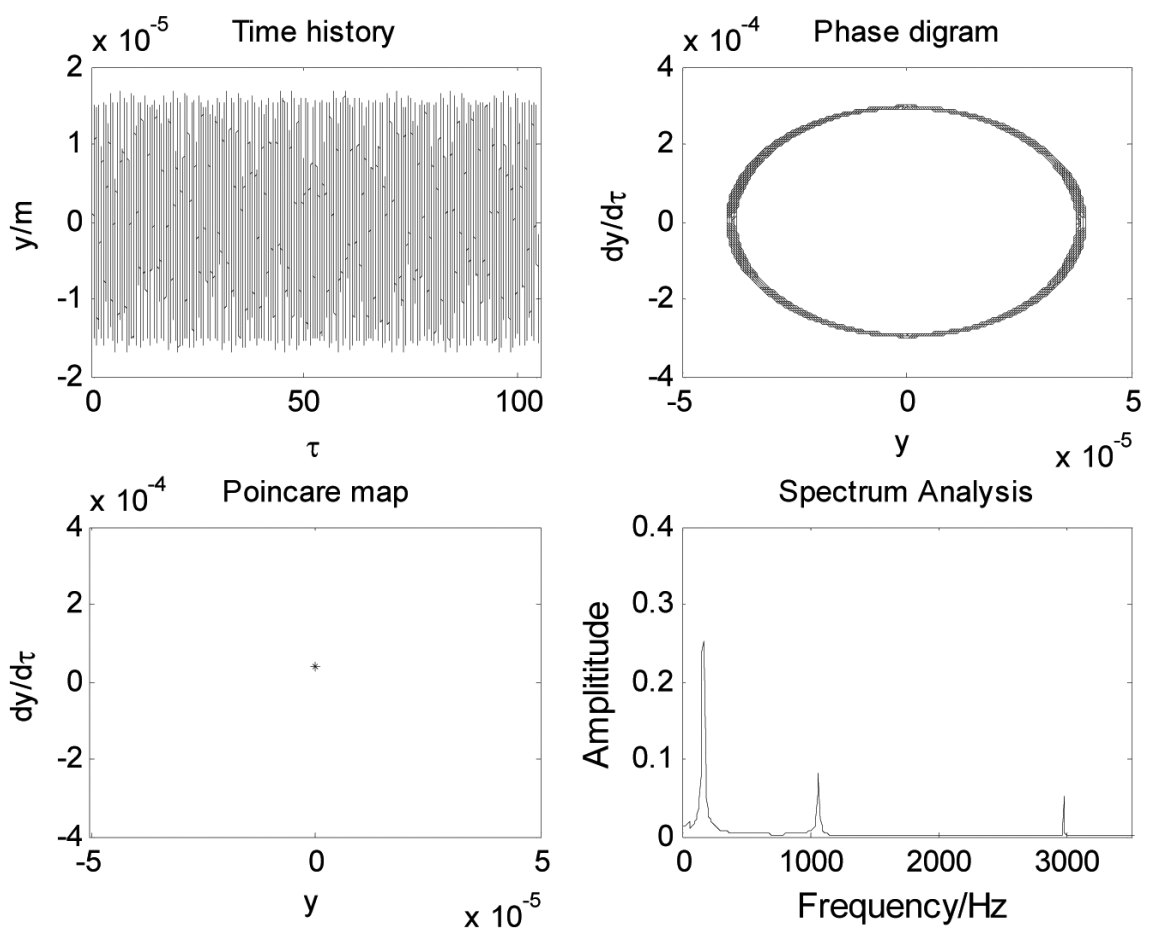

(a) $n=2000 \mathrm{rpm}, \Delta=0.1 \mathrm{~mm}$
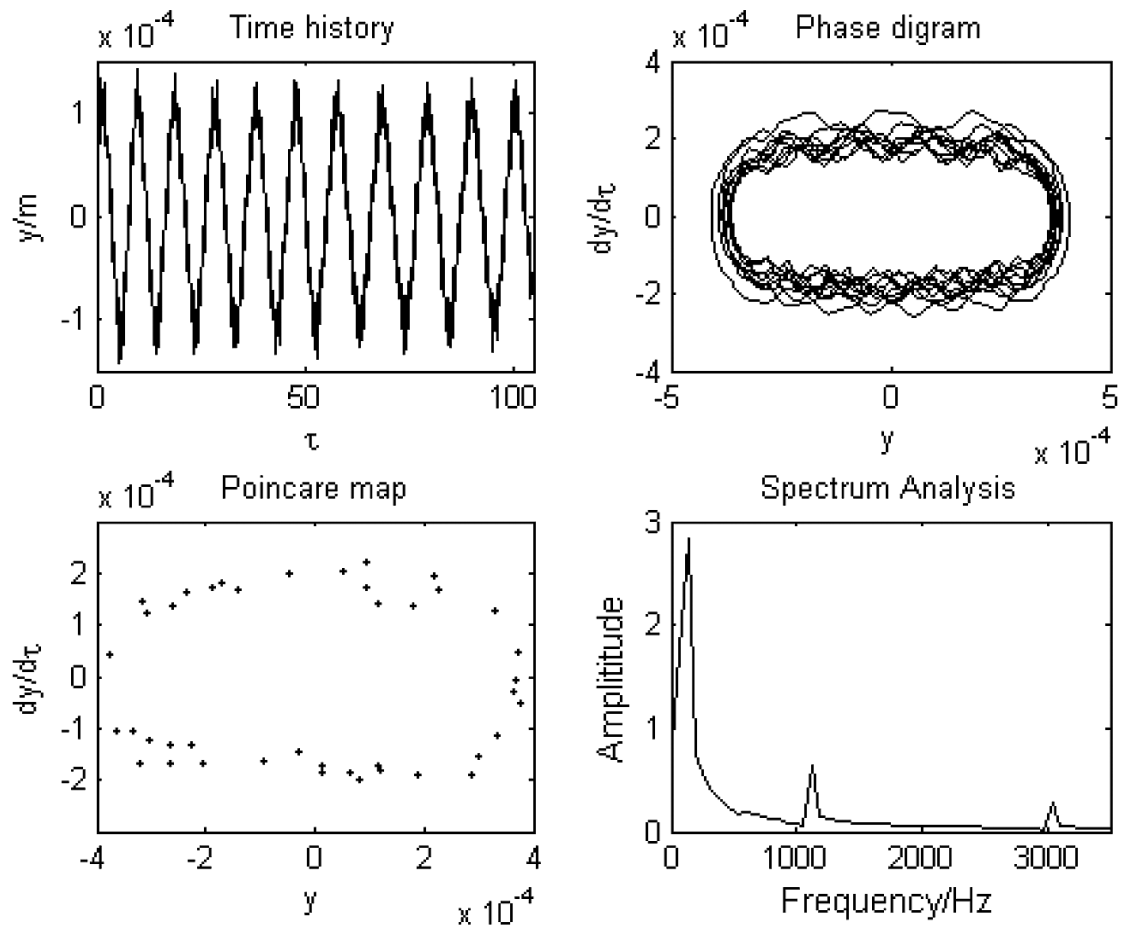

(b) $n=13000 \mathrm{rpm}, \Delta=0.1 \mathrm{~mm}$.

Fig. 4. Responses of the system at rotating speed $2000 \mathrm{rpm}$ and $13000 \mathrm{rpm}$. 


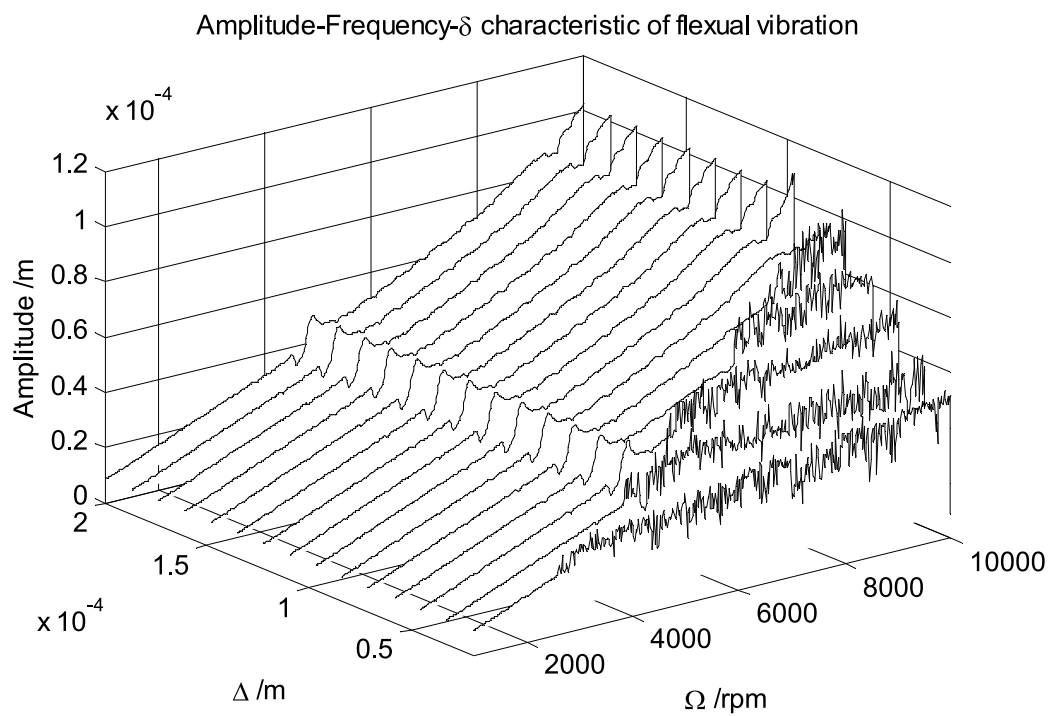

Fig. 5. Relationship of the amplitude of the flexural vibration with rotational speed and gap.

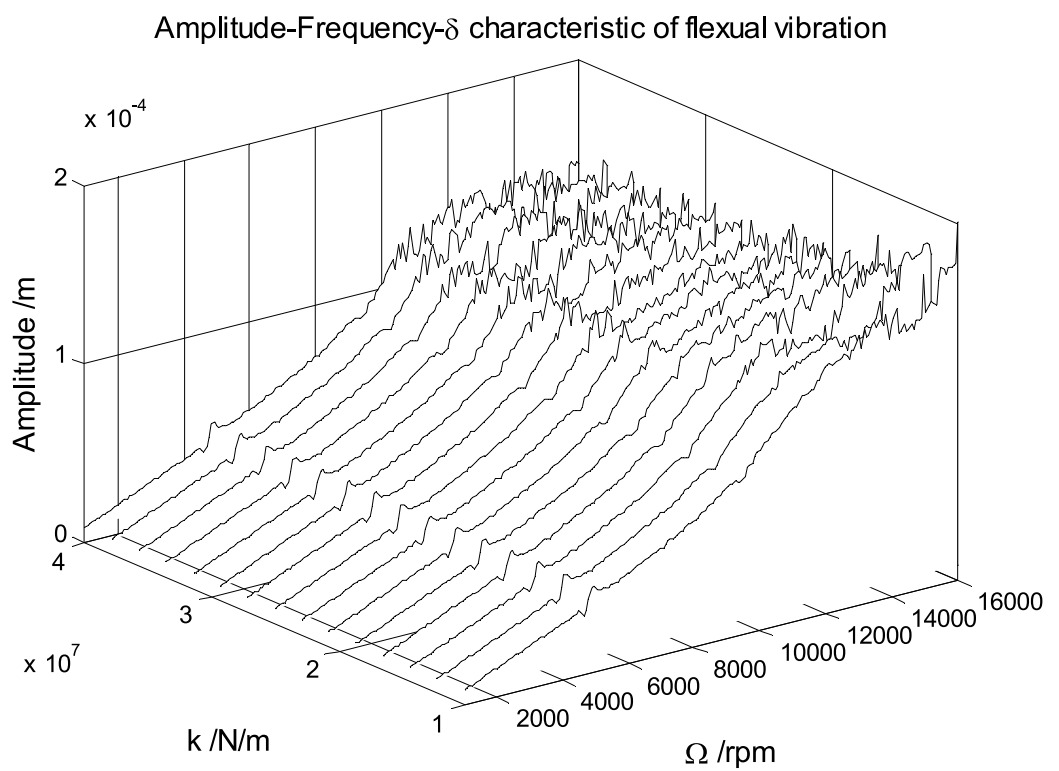

Fig. 6. Relationship of the amplitude of the flexural vibration with, rotational speed and stiffness.

from $1000 \mathrm{rpm}$ to $10000 \mathrm{rpm}$. It can be observed from Fig. 5 that for a given tip gap, there exist a corresponding rotational speed from which the collision between the blade and the tip will happen. If the rotational speed increases further, the variation of the tip gap has a great effect on the response amplitude of the blade end. In this case, the smaller the tip gap is and the better vibration reduction effects will be obtained. Moreover the amplitude of the blade vibration is invariable when the tip gap $\Delta$ is greater than $0.1 \mathrm{~mm}$ at a certain speed because of non-collision. Therefore in order to greatly suppress the vibration of the aero-engine rotor-blade, the tip gap $\Delta$ should be made as small as possible.

Figure 6 presents the relationship of the response amplitude versus the elastic coefficient between the stiffness of the tip $k$ and the rotational speed of the blade. The elastic coefficient $k$ varies from $1 \times 10^{7} \mathrm{~N} / \mathrm{m}$ to $4 \times 10^{7} \mathrm{~N} / \mathrm{m}$ and the rotational speed varies from $1000 \mathrm{rpm}$ to $10000 \mathrm{rpm}$. As shown in Fig. 6 when the rotational speed is greater 
than $2410 \mathrm{rpm}$, the variation of the elastic coefficient $k$ has a little effect on the response amplitude of the blade end. When the elastic coefficient $k$ is increscent from $1 \times 10^{7} \mathrm{~N} / \mathrm{m}$ to $4 \times 10^{7} \mathrm{~N} / \mathrm{m}$ the amplitude of the blade end is somewhat reduced. In other words, the increase of the elastic coefficient $k$ will be appropriate to get the better vibration reduction effects.

\section{Conclusions}

The damped vibration of blades with tips has been formulated due to combined motions of impact and friction between blades and tips, and the dry friction model has been used to simulate the effect of frictional constraint at the shroud interfaces. Some available results related to damped vibration characteristics of the blade with regard to the rotational speed, the tip gap $\Delta$, and elastic coefficient $k$ are numerically obtained respectively. The main conclusions obtained in this work are as follows:

(1) The response amplitude of the flexural vibration of the blade is getting larger with the increasing of the rotational speed. When the rotational speed is less than $2410 \mathrm{rpm}$ and the displacement of the blade end is not large enough to collide with the tip, i.e., $v(l)<\Delta(=0.02 \mathrm{~mm})$, the variation of the tip gap $\Delta$ has no effect on the amplitude of the blade end.

(2) When the rotational speed is greater than $2410 \mathrm{rpm}$, the amplitude of the blade end is greater than the initial gap $\Delta=0.02 \mathrm{~mm}$. In this case, the blade end will collide with the tip and the collision will decrease the vibration of the blade-tip system.

(3) The tip gap has a great effect on the response amplitude of the blade end. In order to suppress the vibration of the rotating blade-tip system, the tip gap should be made as small as possible. The elastic coefficient $k$ has little effect on the amplitude of the blade end.

The calculation method, the basic analysis approach and the important conclusions provided in this paper may be available for reference in the designing, development and modification of aero-engine turbo.

\section{Acknowledgements}

The authors acknowledge the supports of the National Natural Science Foundation of China under Grant No. 10772056 and No. 90816002, and the Harbin Science and Technology Innovative Foundation under Grant No. 2007RFLXG009.

\section{References}

[1] J.P. Den Hartog, Forced vibrations with combined coulomb and viscous friction, Transactions of the American Society of Mechanical Engineers 53(1) (1931), 107-115.

[2] C.K. Gordon and G. Yeh, Forced vibration of a two-degree of freedom system with combined coulomb and viscous damping, Journal of Acoustical Society of America 39(1) (1966), 14-24.

[3] C. Pierre, A.A. Ferri and E.H. Dowell, Multi-harmonic analysis of dry friction damped systems using an incremental harmonic balance method, Journal of Applied Mechanics 52(4) (1985), 958-964.

[4] A. Muszynska and J. Dig, Bladed disk dynamics investigated by a discrete model: effects of traveling wave excitation friction and mistuning, Proceedings of the Machinery Vibration Monitoring and Analysis Meeting (1982), 33-49.

[5] A.A. Ferri and E.H. Dowell, Frequency domain solutions to multi-degree of freedom, dry friction damped systems, Journal of Sound and Vibration 124 (1988), 207-224.

[6] J.H. Wang and W.K. Chen, Investigation of the vibration of a blade with friction damper by hbm, Transactions of the ASME 115 (1993), 294-299.

[7] K.Y. Sanliturk, M. Imregun and D.J. Ewins, Harmonic balance vibration analysis of turbine blades with friction dampers, Journal of Vibration and Acoustics 119 (1997), 96-103.

[8] K. Popp, L. Panning and W. Sextro, Vibration damping by friction forces: Theory and applications, Journal of Vibration and Control $\mathbf{9}$ (2003), 419-448.

[9] W.D. Iwan, The dynamic response of bilinear hysteretic systems, California Institute of Technology, 1966. 
[10] C.H. Menq and J.H. Griffin, A comparison of transient and steady state finite element analyses of the forced response of a frictionally damped beam, Journal of Vibration, Acoustics, Stress, and Reliability in Design 107 (1985), 19-25.

[11] J.H. Griffin, A review of friction damping of turbine blade vibration, International Journal of Turbo and Jet Engines 7 (1990), $297-307$.

[12] Q. Ding and X. Zhou, Analytical method determining resonant response of blade with dry friction damper, Transactions of Tianjin University 13(4) (2007), 291-296.

[13] Q. Ding and Y. Chen, Analyzing resonant response of a system with dry friction damper using an analytical method, Journal of Vibration and Control 14(8) (2008), 1111-1123.

[14] G.F. Nan, X.M. Ren, S.W. He and Y.F. Yang, Damped vibration characteristics of blades with tips of an aero-engine, Journal of Vibration and Shock 28(7) (2009), 135-138.

[15] C.H. Menq, J. Bielak and J.H. Griffin, The influence of micro-slip on vibratory response, part 1: a new theoretical model, Journal of Sound and Vibration 107 (1986), 279-293.

[16] C.H. Menq, P. Chidamparam and J.H. Griffin, Friction damping of two - dimensional motion and its application in vibration and control, Journal of Sound and Vibration 144 (1991), 427-447.

[17] J.H. Griffin and C.H. Menq, Friction damping of circular motion and its application to vibration control, Journal of Vibration and Acoustics 113(2) (1991), 225-229.

[18] C.H. Menq and B.D. Yang, Nonlinear spring resistance and friction damping of frictional constraint having two-dimensional motion, Journal of Sound and Vibration 217 (1998), 127-143.

[19] B.D. Yang and C.H. Menq, Modeling of friction contact and its application to the design of shroud contact, Journal of Engineering for Gas Turbines and Power 119 (1997), 958-963.

[20] B.D. Yang, J.J. Chen and C.H. Menq, Prediction of resonant response of shrouded blades with three-dimensional shroud constraint, Journal of Engineering for Gas Turbines and Power 121 (1999), 523-529.

[21] J.J. Chen and C.H. Menq, Periodic response of blades having three-dimensional nonlinear shroud constraints, Journal of Engineering for Gas Turbines and Power 123 (2001), 901-909.

[22] E. Ciğeroğlu and H. Nevzat Özgüven, nonlinear vibration analysis of bladed disks with dry friction dampers, Journal of Sound and Vibration 295 (2006), 1028-1043. 

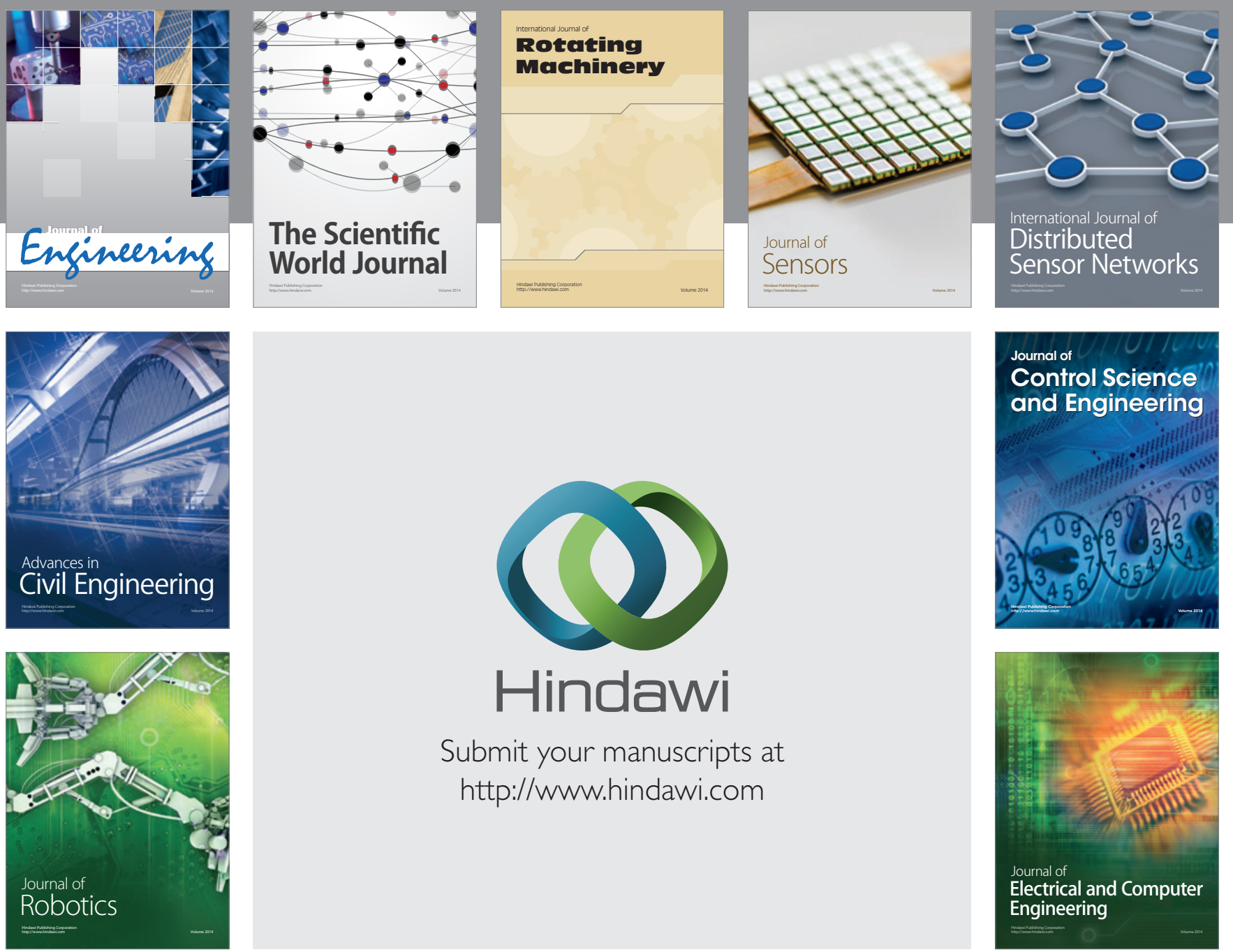

Submit your manuscripts at

http://www.hindawi.com
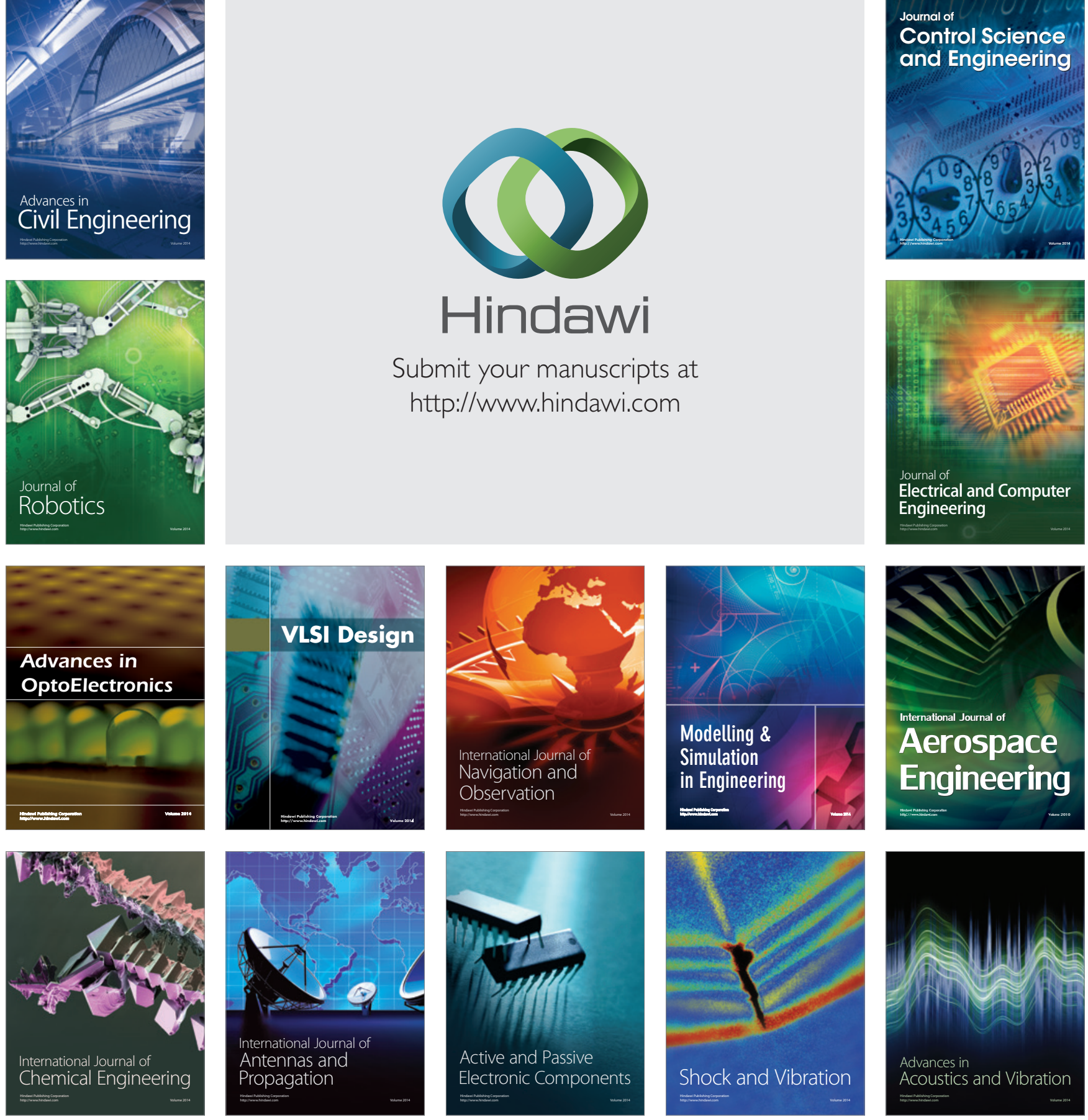